\title{
Wigner Paths Method in Quantum Transport with Dissipation
}

\author{
P. BORDONE*, A. BERTONI, R. BRUNETTI and C. JACOBONI \\ Istituto Nazionale per la Fisica della Materia, Dipartimento di Fisica, Via Campi 213/A, I-41100 Modena, Italy
}

\begin{abstract}
The concept of Wigner paths in phase space both provides a pictorial representation of the quantum evolution of the system of interest and constitutes a useful tool for numerical solutions of the quantum equation describing the time evolution of the system. A Wigner path is defined as the path followed by a "simulative particle" carrying a $\delta$-contribution of the Wigner function through the Wigner phase-space, and is formed by ballistic free flights separated by scattering processes (both scattering with phonons and with an arbitrary potential profile can be included), as for the case of semiclassical particles. Thus, the integral transport equation can be solved by a Monte Carlo technique by means of simulative particles following classical trajectories, in complete analogy to the "Weighted Monte Carlo" solution of the Boltzmann equation in the integral form.
\end{abstract}

Keywords: Wigner paths; Quantum transport; Quantum Monte Carlo procedure

\section{INTRODUCTION}

The Wigner function (WF) was introduced as an extension of the concept of distribution function to the quantum case, and it constitutes the more direct link between the quantum density matrix and the "classical" description of the evolution of the system in phase space through a distribution $f(\mathbf{r}, \mathbf{k}, t)$. It is known from the literature [1] that Wigner trajectories (WT's) in phase space can be defined by modified Hamilton equations, which are very useful for a pictorial representation of the quantum evolution of the system of interest. For quantum systems in stationary states WT's are the
"equi-WF" curves. On the other hand, there are several difficulties in the theory of WT's that make them of little utility for most cases of practical interest. WT's can differ from the corresponding classical trajectories even when the classical analogue of the quantum system exists (they do coincide only in very few special cases [2]). The Liouville theorem does not hold for the WF at singularities in the effective potential entering the WF evolution equation. At these points WT's can be created or destroyed. More important, the quantum force which appears in the effective Hamilton equations depends on the state of the system. Thus many WT's are defined for a single

*Corresponding author. Tel.: + 39-059-205-5277, Fax: +39-059-367488. 
initial phase-space point, each of them for any given quantum state of the system. It should be noticed that, due to the above limitations, such approach has produced no results for the case of carrier-phonon coupling, which is of fundamental importance for the study of the transport properties in many mesoscopic real structures.

In this paper we discuss the concept of Wigner paths (WP's), recently introduced by the authors $[3,4]$, which overcome the theoretical problems discussed above, and which can be used also when the coupling of the carrier with phonons and/or with an arbitrary potential profile is present. WP's are based on the linearity of the evolution equation for the WF and are independent of the quantum state of the system; each WP describes the evolution of a $\delta$-like contribution to the WF, and contains "ballistic flights" and "scattering events" in strict analogy with carriers paths in semiclassical transport.

The WP's can be derived following two different but equivalent analytical approaches. They are both described, for a deeper understanding of the physical meaning of the WP's concept, in Section 2. In Section 3 the theory is applied to some specific cases of interest. First the evolution of a Gaussian WF in a quantum well with infinite potential barriers is discussed. Then a Monte Carlo (MC) solution of the WF evolution equation is introduced and used to perform a comparison between quantum and classical treatement of the scattering mechanisms. The generalization of the WP's approach to the case of a two-time WF is breafly presented.

\section{THEORY}

The general system we are interested in is an ensamble of independent electrons subject to a constant and uniform accelerating field $\mathbf{E}$, to a structure potential $V(\mathbf{r})$, and to the interaction with phonons. The Hamiltonian of the system is given by

$$
\mathbf{H}=\mathbf{H}_{0}+V(\mathbf{r})+V_{f}(\mathbf{r})+\mathbf{H}_{p}+\mathbf{H}_{e-p}
$$

where

$$
\begin{aligned}
\mathbf{H}_{0} & =-\frac{\hbar^{2}}{2 m} \nabla_{\mathbf{r}}^{2}, \\
V_{f}(\mathbf{r}) & =-e \mathbf{E} \cdot \mathbf{r}, \\
\mathbf{H}_{p} & =\sum_{\mathbf{q}} \mathbf{b}_{\mathbf{q}}^{\dagger} \mathbf{b}_{\mathbf{q}} \hbar \omega_{\mathbf{q}},
\end{aligned}
$$

and

$$
\mathbf{H}_{e-p}=\sum_{\mathbf{q}} i \hbar F(\mathbf{q})\left(\mathbf{b}_{\mathbf{q}} e^{i \mathbf{q r}}-\mathbf{b}_{\mathbf{q}}^{\dagger} e^{i \mathbf{q r}}\right),
$$

are, respectively, the free electron term (with $m$ electron effective mass), the electric field term, the Hamiltonian for the free phonon system and the electron-phonon interaction term. In the above expressions $\mathbf{b}_{\mathbf{q}}$ and $\mathbf{b}_{\mathbf{q}}^{\dagger}$ are the annihilation and creation operators for the phonon mode $\mathbf{q}$ with frequency $\omega_{\mathbf{q}}$ and $F(\mathbf{q})$ is a function depending on the type of phonon scattering analyzed.

The generalized WF [5] for an electron - phonon system is:

$$
\begin{aligned}
& f_{w}\left(\mathbf{r}, \mathbf{p},\left\{n_{\mathbf{q}}\right\},\left\{n_{\mathbf{q}}^{\prime}\right\}, t\right) \\
& \quad=\int d \mathbf{r}^{\prime} e^{-i \mathbf{p} \cdot \mathbf{r}^{\prime} / \hbar}\left\langle\mathbf{r}+\mathbf{r}^{\prime} / 2, n_{\mathbf{q}}|\rho(t)| \mathbf{r}-\mathbf{r}^{\prime} / 2,\left\{n_{\mathbf{q}}^{\prime}\right\}\right\rangle
\end{aligned}
$$

where $\rho$ is the density operator of the electronphonon system. Tracing over the phonon coordinates provides the WF representative of the electron system [6].

Using this general frame WP's can be obtained following two different analytical approaches.

\subsection{Transfer Coefficients Approach}

For any given basis $\{|\varphi n\rangle\}$ in the space of the electron states the coefficients [7]

$$
\begin{aligned}
f_{n n^{\prime}}(\mathbf{r}, \mathbf{p})= & \int d \mathbf{r}^{\prime} e^{-i \mathbf{p} \cdot \mathbf{r}^{\prime} / \hbar} \\
& \times\left\langle\mathbf{r}+\mathbf{r}^{\prime} / 2 \mid \varphi_{n}\right\rangle\left\langle\varphi_{n^{\prime}} \mid \mathbf{r}-\mathbf{r}^{\prime} / 2\right\rangle .
\end{aligned}
$$

they constitute a unitary transformation and connect the generalized WF to the density matrix $\rho\left(n\left\{n_{\mathbf{q}}\right\}, n^{\prime}\left\{n_{\mathbf{q}}^{\prime}\right\}, t\right)$ and viceversa. 
Using the Liouville equation for the density matrix in the interaction picture, it is possible to derive the equation for the corresponding WF. After a formal integration with respect to time and a transformation back to the Schrödinger picture, the following integral equation for the WF is obtained [3]:

$$
\begin{aligned}
f_{w}\left(\mathbf{r}, \mathbf{p},\left\{n_{\mathbf{q}}\right\},\left\{n_{\mathbf{q}}^{\prime}\right\}, t\right) \\
=\frac{1}{h^{3}} \sum_{n n^{\prime}} f_{n n^{\prime}}(\mathbf{r}, \mathbf{p}) e^{-i\left(\omega\left(n\left\{n_{\mathbf{q}}\right\}\right)-\omega\left(n^{\prime}\left\{n_{\mathbf{q}}^{\prime}\right\}\right)\right)\left(t-t_{\circ}\right)} \\
\quad \int d \mathbf{r}^{\prime} \int d \mathbf{p}^{\prime} f_{n n^{\prime}}^{*}\left(\mathbf{r}^{\prime}, \mathbf{p}^{\prime}\right) f_{w}\left(\mathbf{r}^{\prime}, \mathbf{p}^{\prime},\left\{n_{\mathbf{q}}\right\},\left\{n_{\mathbf{q}}^{\prime}\right\}, t_{\circ}\right) \\
+\frac{1}{h^{3}} \sum_{n n^{\prime}} f_{n n^{\prime}}(\mathbf{r}, \mathbf{p}) \int_{t_{\circ}}^{t} d t^{\prime} e^{-i\left(\omega\left(n\left\{n_{\mathbf{q}}\right\}\right)-\omega\left(n^{\prime}\left\{n_{\mathbf{q}}^{\prime}\right\}\right)\right)\left(t-t^{\prime}\right)} \\
\quad \times \sum_{m m_{q}} \int d \mathbf{r}^{\prime} \int d \mathbf{p}^{\prime}\left\{\mathcal{H}^{\prime}\left(n\left\{n_{\mathbf{q}}\right\}, m\left\{m_{\mathbf{q}}\right\}\right)\right. \\
\quad \times f_{m n^{\prime}}^{*}\left(\mathbf{r}^{\prime}, \mathbf{p}^{\prime}\right) f_{w}\left(\mathbf{r}^{\prime}, \mathbf{p}^{\prime},\left\{m_{\mathbf{q}}\right\},\left\{n_{\mathbf{q}}^{\prime}\right\}, t^{\prime}\right)-f_{n m}^{*}\left(\mathbf{r}^{\prime}, \mathbf{p}^{\prime}\right) \\
\quad \times f_{w}\left(\mathbf{r}^{\prime}, \mathbf{p}^{\prime},\left\{n_{\mathbf{q}}\right\},\left\{m_{\mathbf{q}}\right\}, t^{\prime}\right) \mathcal{H}^{\prime}\left(m\left\{m_{\mathbf{q}}\right\}, n^{\prime}\left\{n_{\mathbf{q}}^{\prime}\right\}\right) \\
=f_{w}^{(\circ)}+\Delta f_{w}
\end{aligned}
$$

where $\mathcal{H}^{\prime}=\left(\mathbf{H}_{\mathbf{e}-\mathbf{p}} / i \hbar\right)$. In Eq. (4) the first term in the r.h.s. describes the ballistic coherent propagation from the initial time $t_{\mathrm{o}}$ to the observation time $t$. The second term describes the contribution of the unknown WF to the "last" interaction vertex at any time $t^{\prime}$ between $t_{\mathrm{o}}$ and $t$ followed by the free propagation from $t^{\prime}$ to $t$. Eq. (4) is written for the entire system and contains also the phonon variables. Moreover it is foundamental to notice that the above integral equation is linear in the unknown WF. This guarantee that if the WF at $t=t_{\mathrm{o}}$ is the sum of several contributions, then each of them will evolve according to Eq. (4), and the solution of the equation at any given time $t>t_{\mathrm{o}}$ will be given by the sum of the single contributions evaluated at the same time.

Even though a point-like electronic WF in phase space cannot represent a real physical system, since it violates the uncertainty principle, we may however start to consider the evolution in time of a $\delta$-like contribution to the $\mathrm{WF}, \delta^{3}\left(\mathbf{r}-\mathbf{r}_{\mathrm{o}}\right) \delta^{3}\left(\mathbf{p}-\mathbf{p}_{\mathrm{o}}\right) f_{P}\left(\left\{n_{\mathbf{q}}\right\},\left\{n_{\mathbf{q}}^{\prime}\right\}\right)$, inside Eq. (4). In absence of phonon coupling the freeelectron Wigner trajectories are the same as for semiclassical particles. In fact, for a plane-wave basis the coefficients $f_{l m}$ are given by

$$
f_{\mathbf{k}_{l} \mathbf{k}_{m}^{\prime}}(\mathbf{r}, \mathbf{p})=\hbar^{3} e^{i\left(\mathbf{k}_{l}-\mathbf{k}_{m}^{\prime}\right) \cdot \mathbf{r}} \delta^{3}\left(\mathbf{p}-\hbar \frac{\left(\mathbf{k}_{l}+\mathbf{k}_{m}^{\prime}\right)}{2}\right)
$$

and the ballistic evolution of the WF as given by the first term on the r.h.s. of Eq. (4) yields:

$$
\begin{aligned}
f_{w}\left(\mathbf{r}, \mathbf{p},\left\{n_{\mathbf{q}}\right\},\left\{n_{\mathbf{q}}^{\prime}\right\}, t\right) \\
=f_{w}\left(\mathbf{r}-\frac{\mathbf{p}}{m}\left(t-t_{\mathbf{o}}\right), \mathbf{p},\left\{n_{\mathbf{q}}\right\},\left\{n_{\mathbf{q}}^{\prime}\right\}, t_{\mathrm{o}}\right) \\
\quad \times e^{-i\left(\omega\left(\left\{n_{\mathbf{q}}\right\}\right)-\omega\left(\left\{n_{\mathbf{q}}^{\prime}\right\}\right)\right)\left(t-t_{\mathbf{o}}\right)}
\end{aligned}
$$

where $\hbar \omega\left(\left\{n_{\mathbf{q}}\right\}\right)$ is the total energy of the phonon bath in the state $\left\{n_{\mathbf{q}}\right\}$. The two factors on the r.h.s. of Eq. (6) describe the free trajectory of the electron and the time evolution of the free-phonon bath, respectively. The above equation can be interpreted as follows: each $\delta$-like contribution to the $\mathrm{WF}$, in absence of external forces, carries its value following a classical path. This means, for example that, if the WF has a wave-packet form, such packet will be "deformed" during the time evolution due to the different paths followed by its $\delta$-like components: representative points in phasespace corresponding to high momentum values move faster than low-momentum representative points [4]. The above result can be generalized to the case of electron Hamiltonians including a constant force or a harmonic potential. Summurizing, we can say that the WF evolves very much like ensambles of semiclassical particles, except for fast varying potentials and scattering dynamics. It is fundamental to notice that this consideration can be crucial for explaining the reliability of the semiclassical model for carrier transport far beyond the expected limits.

Concerning the scattering dynamics it should be noticed that similar trajectories exist, as long as a single interaction diagram is considered without time integrations. In particular, taking into account phonon scattering, we may select the two times of the scattering vertices and a sigle mode $\mathbf{q}$ of the phonon interacting with the electron, and each $\delta$-contribution still remains a $\delta$. With the potential, we have to select a scattering time and a 
transferred momentum, and again a $\delta$-like WF keeps its $\delta$-character. These considerations allow us to define a WP [3] as the path followed by a "simulative particle".

\subsection{Path Variables Approach}

If the time derivative of Eq. (2) is taken and the Liouville-von Neumann equation for the evolution of the density matrix is used, we find

$$
\begin{aligned}
& \frac{\partial}{\partial t} f_{w}\left(\mathbf{r}, \mathbf{p},\left\{n_{\mathbf{q}}\right\},\left\{n_{\mathbf{q}}^{\prime}\right\}, t\right) \\
& \quad=\frac{1}{i \hbar} \int d \mathbf{s} e^{-(i / \hbar) \mathbf{p} \cdot \mathbf{s}}\left\langle\mathbf{r}+\frac{\mathbf{s}}{2},\left\{n_{\mathbf{q}}\right\}|[\mathbf{H}, \rho(t)]| \mathbf{r}-\frac{\mathbf{s}}{2},\left\{n_{\mathbf{q}}^{\prime}\right\}\right\rangle
\end{aligned}
$$

Using the Hamiltonian given in Eq. (1) the r.h.s. of the above equation can be written as the sum of five terms. We will consider each of them separately in a schematic way (the full derivation is given in Ref. [4]).

The term containing $\mathbf{H}_{0}$ leads to a term containing the space derivative of the distribution:

$$
\begin{gathered}
\frac{1}{i \hbar} \int d \mathbf{s} e^{(-i / \hbar) \mathbf{p} \cdot \mathbf{s}}\left\langle\mathbf{r}+\frac{\mathbf{s}}{2},\left\{n_{\mathbf{q}}\right\}\left|\left[\mathbf{H}_{0}, \rho(t)\right]\right| \mathbf{r}-\frac{\mathbf{s}}{2},\left\{n_{q}^{\prime}\right\}\right\rangle \\
\quad=-\frac{\mathbf{p}}{m} \cdot \nabla_{\mathbf{r}} f_{w}\left(\mathbf{r}, \mathbf{p},\left\{n_{\mathbf{q}}\right\},\left\{n_{\mathbf{q}}^{\prime}\right\}, t\right)
\end{gathered}
$$

The term due to the potential profile leads to:

$$
\begin{gathered}
\frac{1}{i \hbar} \int d \mathbf{s} e^{(-i / \hbar) \mathbf{p} \cdot \mathbf{s}}\left\langle\mathbf{r}+\frac{\mathbf{s}}{2},\left\{n_{\mathbf{q}}\right\}|[V(\mathbf{r}), \rho(t)]| \mathbf{r}-\frac{\mathbf{s}}{2},\left\{n_{\mathbf{q}}^{\prime}\right\}\right\rangle \\
=\int d \mathbf{p} \mathcal{V}_{w}\left(\mathbf{r}, \mathbf{p}-\mathbf{p}^{\prime}\right) f_{w}\left(\mathbf{r}, \mathbf{p}^{\prime},\left\{n_{\mathbf{q}}\right\},\left\{n_{\mathbf{q}}^{\prime}\right\}, t\right)
\end{gathered}
$$

where the transfer function $\mathcal{V}_{w}$ is defined by

$$
\mathcal{V}_{w}(\mathbf{r}, \mathbf{p})=\frac{1}{h^{3}} \int d \mathbf{s} e^{(-i / \hbar) \mathbf{p} \cdot \mathbf{s}} \frac{1}{i \hbar}\left[V\left(\mathbf{r}+\frac{\mathbf{s}}{2}\right)-V\left(\mathbf{r}-\frac{\mathbf{s}}{2}\right)\right] .
$$

The term due to a constant uniform electric field leads to:

$$
\begin{gathered}
\frac{1}{i \hbar} \int d \mathbf{s} e^{(-i / \hbar) \mathbf{p} \cdot \mathbf{s}}\left\langle\mathbf{r}+\frac{\mathbf{s}}{2},\left\{n_{\mathbf{q}}\right\}\left|\left[V_{f}(\mathbf{r}), \rho(t)\right]\right| \mathbf{r}-\frac{\mathbf{s}}{2},\left\{n_{\mathbf{q}}^{\prime}\right\}\right\rangle \\
=e \mathbf{E} \cdot \nabla_{\mathbf{p}} f_{w}\left(\mathbf{r}, \mathbf{p},\left\{n_{\mathbf{q}}\right\},\left\{n_{\mathbf{q}}^{\prime}\right\}, t\right) .
\end{gathered}
$$

the same result would be obtained for a harmonic potential.

The term describing the free evolution of the phonon bath may be written as

$$
\begin{array}{r}
\frac{1}{i \hbar} \int d \mathbf{s} e^{(-i / \hbar) \mathbf{p} \cdot \mathbf{s}}\left\langle\mathbf{r}+\frac{\mathbf{s}}{2},\left\{n_{\mathbf{q}}\right\}\left|\left[\boldsymbol{H}_{p}, \rho(t)\right]\right| \mathbf{r}-\frac{\mathbf{s}}{2},\left\{n_{\mathbf{q}}^{\prime}\right\}\right\rangle \\
=\frac{1}{i \hbar}\left(\varepsilon\left(\left\{n_{\mathbf{q}}\right\}\right)-\varepsilon\left(\left\{n_{\mathbf{q}}^{\prime}\right\}\right)\right) f_{W}\left(\mathbf{r}, \mathbf{p},\left\{n_{\mathbf{q}}\right\},\left\{n_{\mathbf{q}}^{\prime}\right\}, t\right),
\end{array}
$$

where

$$
\varepsilon\left(\left\{n_{\mathbf{q}}\right\}\right)=\sum_{\mathbf{q}} n_{\mathbf{q}} \hbar \omega_{\mathbf{q}}
$$

is the energy of the phonon state $\left\{n_{\mathbf{q}}\right\}$.

The term due to electron-phonon interaction gives rise to four terms:

$$
\begin{aligned}
\frac{1}{i \hbar} \int d \mathbf{s} & e^{(-i / \hbar) \mathbf{p} \cdot \mathbf{s}}\left\langle\mathbf{r}+\frac{\mathbf{s}}{2},\left\{n_{\mathbf{q}}\right\}\left|\left[H_{e-p}, \rho(t)\right]\right| \mathbf{r}-\frac{\mathbf{s}}{2},\left\{n_{\mathbf{q}}^{\prime}\right\}\right\rangle \\
= & \sum_{\mathbf{q}^{\prime}} F\left(\mathbf{q}^{\prime}\right)\left\{e^{i \mathbf{q}^{\prime} \mathbf{r}} \sqrt{n_{\mathbf{q}^{\prime}}+1} f_{W}\left(\mathbf{r}, \mathbf{p}-\frac{\hbar \mathbf{q}^{\prime}}{2},\left\{n_{1}, \ldots, n_{\mathbf{q}^{\prime}}+1, \ldots\right\},\left\{n_{\mathbf{q}}^{\prime}\right\}, t\right)\right. \\
& -e^{-i \mathbf{q}^{\prime} \mathbf{r}} \sqrt{n_{\mathbf{q}^{\prime}}} f_{W}\left(\mathbf{r}, \mathbf{p}+\frac{\hbar \mathbf{q}^{\prime}}{2},\left\{n_{1}, \ldots, n_{\mathbf{q}^{\prime}}-1, \ldots\right\},\left\{n_{\mathbf{q}}^{\prime}\right\}, t\right) \\
& -e^{i \mathbf{q}^{\prime} \mathbf{r}} \sqrt{n_{\mathbf{q}^{\prime}}^{\prime}} f_{W}\left(\mathbf{r}, \mathbf{p}+\frac{\hbar \mathbf{q}^{\prime}}{2},\left\{n_{\mathbf{q}}\right\},\left\{n_{1}^{\prime}, \ldots, n_{\mathbf{q}^{\prime}}^{\prime}-1, \ldots\right\}, t\right) \\
& \left.+e^{-i \mathbf{q}^{\prime} \mathbf{r}} \sqrt{n_{\mathbf{q}^{\prime}}^{\prime}}+1 f_{W}\left(\mathbf{r}, \mathbf{p}-\frac{\hbar \mathbf{q}^{\prime}}{2},\left\{n_{\mathbf{q}}\right\},\left\{n_{1}^{\prime}, \ldots, n_{\mathbf{q}^{\prime}}^{\prime}+1, \ldots\right\}, t\right)\right\} .
\end{aligned}
$$


The matrix elements in the above equation contain two different sets of phonon occupation numbers $\left\{n_{\mathbf{q}}\right\}$ and $\left\{n_{\mathbf{q}}^{\prime}\right\}$. Each term in the right hand side of Eq. (14) represents a phonon interaction event (vertex) that changes only one set, increasing or decreasing the phonon occupation number of mode $\mathbf{q}^{\prime}$ by one unity and changing the electron momentum by $(\hbar \mathbf{q} / 2)$.

From the above results the following equation is obtained:

$$
\begin{aligned}
\left(\frac{\partial}{\partial t}+\frac{\mathbf{p}}{m}\right. & \left.\cdot \nabla_{r}-e \mathbf{E} \cdot \nabla_{p}\right) f_{W}\left(\mathbf{r}, \mathbf{p},\left\{n_{\mathbf{q}}\right\},\left\{n_{\mathbf{q}}^{\prime}\right\}, t\right) \\
= & \frac{1}{i \hbar}\left(\varepsilon\left(\left\{n_{\mathbf{q}}\right\}\right)-\varepsilon\left(\left\{n_{\mathbf{q}}^{\prime}\right\}\right)\right) f_{W}\left(\mathbf{r}, \mathbf{p},\left\{n_{\mathbf{q}}\right\},\left\{n_{\mathbf{q}}^{\prime}\right\}, t\right) \\
& +\int d \mathbf{p}^{\prime} \mathcal{V}_{W}\left(\mathbf{r}, \mathbf{p}^{\prime}-\mathbf{p}\right) f_{W}\left(\mathbf{r}, \mathbf{p}^{\prime},\left\{n_{\mathbf{q}}\right\},\left\{n_{\mathbf{q}}^{\prime}\right\}, t\right) \\
& +\Xi\left(\mathbf{r}, \mathbf{p},\left\{n_{\mathbf{q}}\right\},\left\{n_{\mathbf{q}}^{\prime}\right\}, t\right)
\end{aligned}
$$

where $\Xi\left(\mathbf{r}, \mathbf{p},\left\{n_{\mathbf{q}}\right\},\left\{n_{\mathbf{q}}^{\prime}\right\}, t\right)$ represents the right hand side of Eq. (14).

The left hand side of Eq. (15) has the same form as the semiclassical Boltzmann equation. Thus path variables can be used in analogy with Chambers formulation of transport. Then, integrating over time, one obtains [8]

$$
\begin{aligned}
f_{W}\left(\mathbf{r}, \mathbf{p},\left\{n_{\mathbf{q}}\right\},\left\{n_{\mathbf{q}}^{\prime}\right\}, t\right) \\
=f_{w}\left(\mathbf{r}-\frac{\mathbf{p}}{m}\left(t-t_{0}\right)+\frac{\mathbf{F}}{2 m}\left(t-t_{0}\right)^{2}, \mathbf{p}-\mathbf{F}\left(t-t_{0}\right),\left\{n_{\mathbf{q}}\right\},\left\{n_{\mathbf{q}}^{\prime}\right\}, t_{0}\right) \\
e^{(-i / f)\left(\varepsilon\left(\left\{n_{\mathbf{q}}\right\}\right)-\varepsilon\left(\left\{n_{\mathbf{q}}^{\prime}\right\}\right)\right)\left(t-t_{0}\right)}+\int_{t_{0}}^{t} d t^{\prime} e^{(-i / \hbar)\left(\varepsilon\left(\left\{n_{\mathbf{q}}\right\}\right)-\varepsilon\left(\left\{n_{\mathbf{q}}^{\prime}\right\}\right)\right)\left(t-t^{\prime}\right)} \\
\times \\
\times\left\{\int d \mathbf{p}^{\prime} \mathcal{V}_{w}\left(\mathbf{r}-\frac{\mathbf{p}}{m}\left(t-t^{\prime}\right)+\frac{\mathbf{F}}{2 m}\left(t-t^{\prime}\right)^{2}, \mathbf{p}^{\prime}-\mathbf{p}+\mathbf{F}\left(t-t^{\prime}\right)\right)\right. \\
\quad \times f_{w}\left(\mathbf{r}-\frac{\mathbf{p}}{m}\left(t-t^{\prime}\right)+\frac{\mathbf{F}}{2 m}\left(t-t^{\prime}\right)^{2}, \mathbf{p}^{\prime},\left\{n_{\mathbf{q}}\right\},\left\{n_{\mathbf{q}}^{\prime}\right\}, t^{\prime}\right) \\
\times \\
\left.\times\left(\mathbf{\Xi}-\frac{\mathbf{p}}{m}\left(t-t^{\prime}\right)+\frac{\mathbf{F}}{2 m}\left(t-t^{\prime}\right)^{2}, \mathbf{p}-\mathbf{F}\left(t-t^{\prime}\right),\left\{n_{\mathbf{q}}\right\},\left\{n_{\mathbf{q}}^{\prime}\right\}, t^{\prime}\right)\right\},
\end{aligned}
$$

with

$$
\mathbf{F}=e \mathbf{E} \text {. }
$$

The above equation shows that the value of the WF in a point $(\mathbf{r}, \mathbf{p})$ at time $t$ comes from three contributions: (a) A ballistic term, equal to the WF's value at time $t_{\mathrm{o}}$ on the trajectory of a classical particle being in $(\mathbf{r}, \mathbf{p})$ at time $t$ and multiplied by a phase factor corresponding to the free evolution of the phonon bath. (b) A term collecting, for each time $t^{\prime}$ and each transferred momentum ( $\left.\mathbf{p}-\mathbf{p}^{\prime}\right)$, contributions from the WF at points of phase space that, after a scattering by the potential $V(\mathbf{r})$, are on the "right" classical trajectory that reaches $(\mathbf{r}, \mathbf{p})$ at time $t$. This term is multiplied by the transfer function $\mathcal{V}_{w}$, acting as a weight factor and by the free phonon evolution pahase factor. (c) A term collecting for each time $t^{\prime}$ the four contributions of the electron-phonon interaction term.

Equation (16) may be iteratively substituted into itself giving a Neumann expansion describing the evolution of the WF by means of simulative particles following classical trajectories and experiencing scatterings with a potential $V(\mathbf{r})$ that change particle's momentum from to $\mathbf{p}^{\prime}$ to $\mathbf{p}$ and with phonons that increase (decrease) by one the number of phonons in $\mathbf{q}$ phonon mode of a single set $\left\{n_{\mathbf{q}}\right\}$ and decrease (increase) particle's momentum by $(\hbar \mathbf{q} / 2)$. The series obtained may be truncated to an arbitrary order and solved by a Monte Carlo technique, sampling the integrals over the scattering times and the momentum transferred by potential or phonons, in complete analogy to the "Weighted Monte Carlo" solution of Boltzmann equation in its integral form [9]. Concerning this point, it should be mentioned that, in the quantum case, different diagrams can contribute to the same WP. Such a contribution is accounted for weighting any specific path with an appropriate multiplicity factor.

\section{APPLICATIONS}

Figure 1 shows the time evolution of the WF of a minimum uncertainity Gaussian wave-packet in a quantum well with infinite potential barriers. 

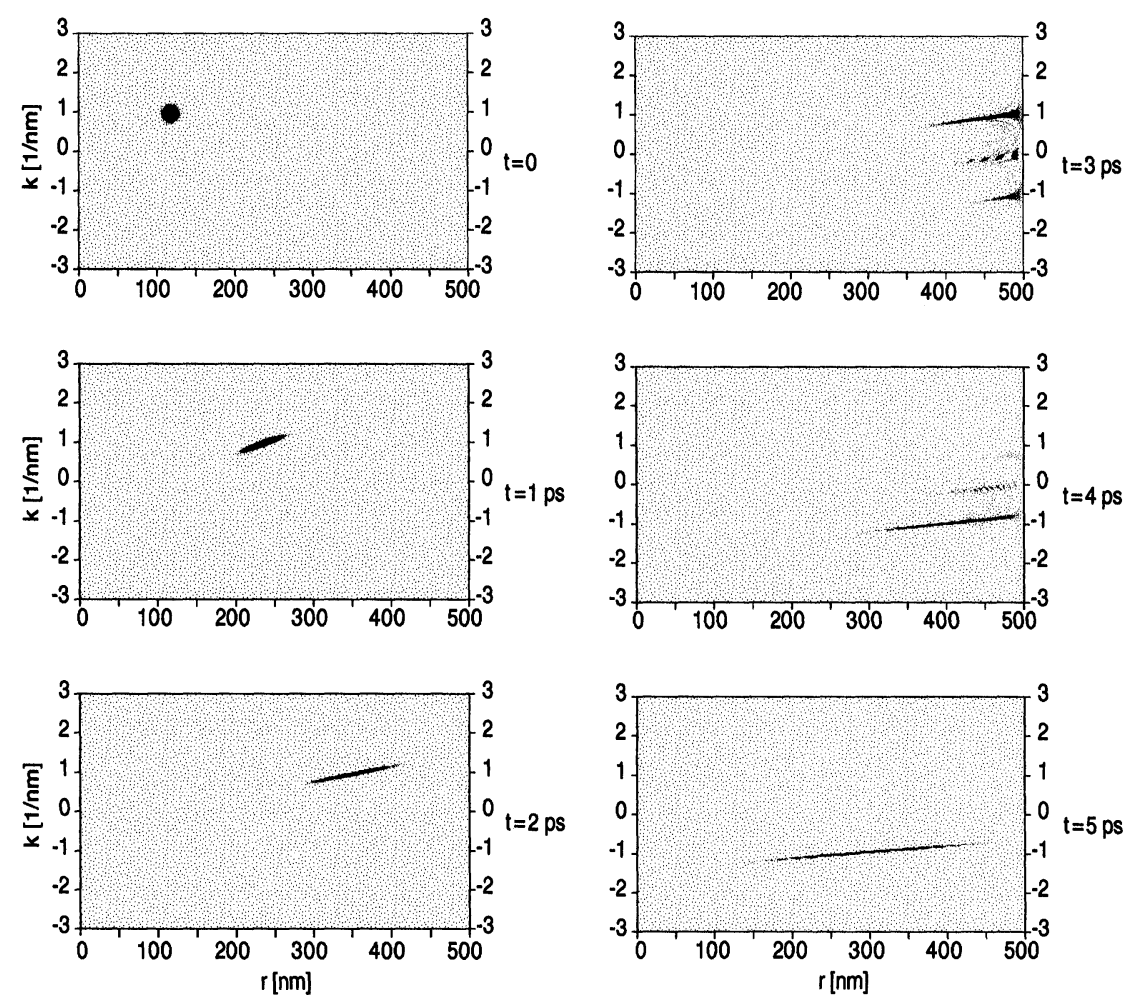

FIGURE 1 Time evolution (given at 6 different instants) of the WF of a minimum uncertainity Gaussian wave-packet in a quantum well with infinite potential barriers. The $f_{\mathrm{W}}$ describes an electron with a gaussian distribution of the initial wave-vector around the value $k_{0}=1 \mathrm{~nm}^{-1}$.

The $f w$ describes an electron with a gaussian distribution of the initial wave-vector around the value $k_{0}=1 \mathrm{~nm}^{-1}$. It is important to note that, before hitting the boundary, the evolution of the WF follows classical trajectories and it is coincident with the evolution of a classical distribution function. Furthermore the wave-packet is deformed in time exactly as for the free particle case. During the collision with the potential barrier interference patterns appear, and the evolution doesn't follow classical trajectories anymore. Finally, for time long enough after the scattering process, the evolution of the WF is found to coincide again with the one of the classical distribution function. The analytical generalization of such a result to an arbitrary form of the WF is at present under development.

Based on the WP's concept, we have developed a MC code in strict analogy with the traditional MC simulation technique used to study semiclassical transport phenomena. In Figure 2 is shown the flow-chart of the quantum MC program, where, for the sake of simplicity, only the scattering with the phonons is considered.

As a first application this quantum simulation program has been used to perform a comparison with the results provided by a code based on a traditional weighted MC algorithm. In particular the effect on transport phenomena of the relaxation of the semiclassical assumption of energy conservation and point-like nature in space/time of the scattering processes have been analyzed. Results obtained for different contributions to the scattering terms of the Wigner and Boltzmann transport equations have been compared. In fact, our rigorous quantum approach allows to analyze in detail real scatterings, virtual 


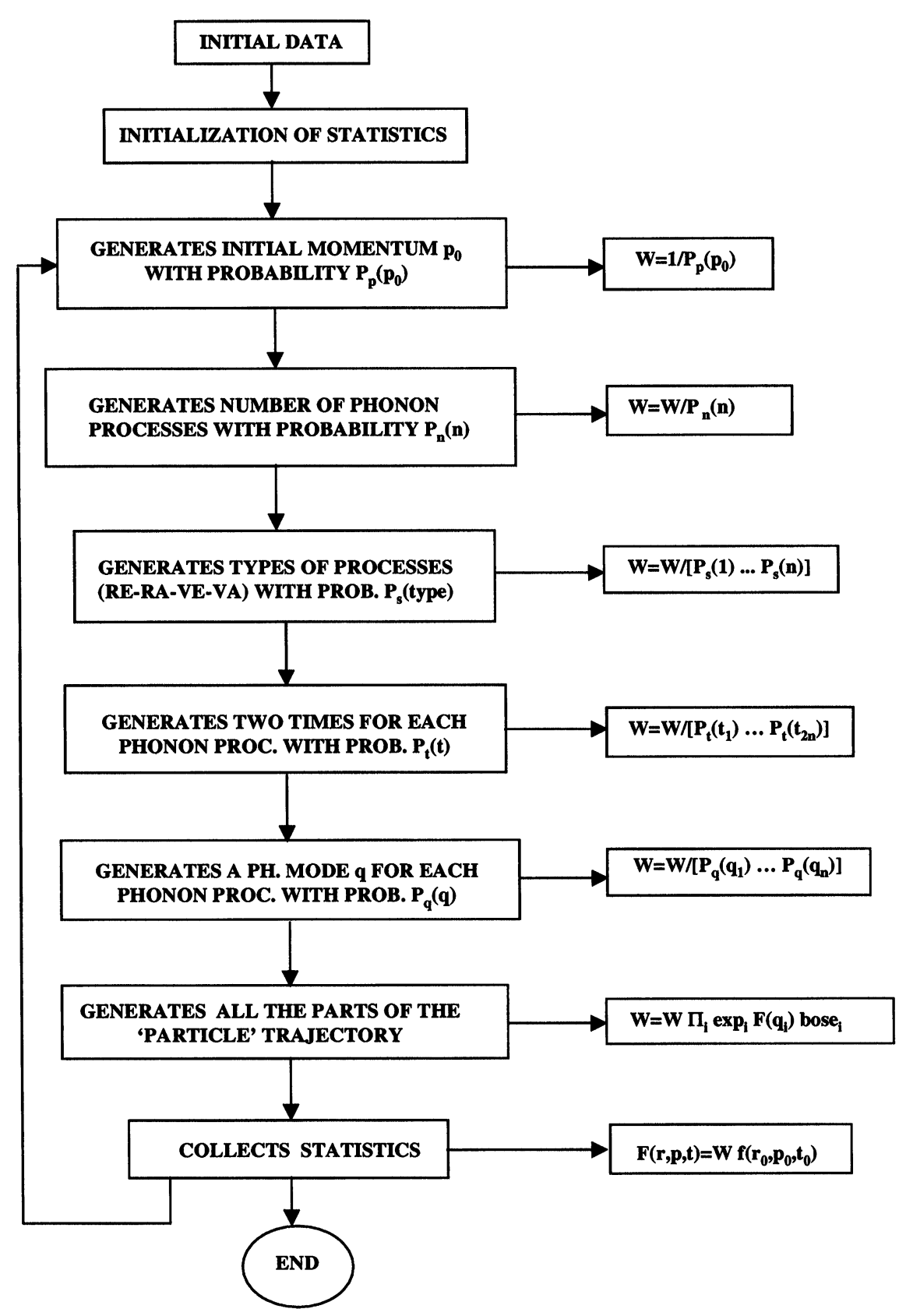

FIGURE 2 Flow-chart of the quantum Monte Carlo code for the case in which only phonon scattering is included.

scatterings, multiple scatterings, and the so called "intra-collisional" field effect.

As an example, we considered the term of the Neumann expansion of the Boltzmann equation describing two in-scattering phonon-emission events and the corresponding terms of the Neumann expansion of the Wigner equation (four-vertices terms associated with two real emissions) [10]. The semiclassical electrons start from a well defined initial state $(z, p z)$ and the 
quantum calculation is performed assuming the same initial condition for a "delta"-like Wigner function, or "simulative particle". Integration is performed over the possible interaction times and phonon modes $\mathbf{q}$ while the observation time is 100 fs. Figure 3 (top) shows the quantum distribution as a function of energy after the two real emissions (continuous line). Since in this case no field is applied, in the classical picture the distribution in Figure 3 would be a delta function at the energy indicated on the figure by the arrow. Contributions coming from separate (dashed line)
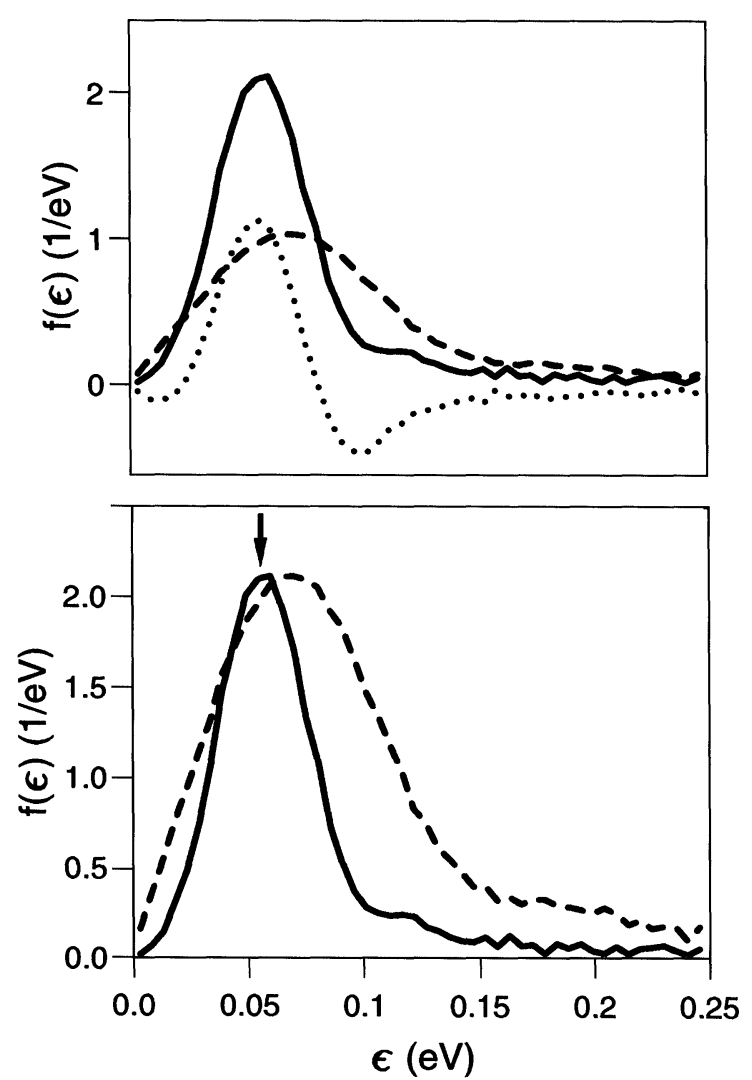

FIGURE 3 Contribution to the energy distribution of the scattering term associated with two real phonon emissions. Top: total contribution (solid line), separate-scattering contribution (dashed-line), multiple-scattering contribution (dotted line). Bottom: comparison between the total contribution (solid line) and the separate-scattering contribution (dashed line) renormalized to the same maximum value. The arrow indicates the energy-conservation value predicted by the semiclassical theory (See Ref. [10]). and from multiple (dotted line) scatterings have been independently evaluated by means of Monte Carlo generations of the correspondind Wigner paths. It is seen that the large energy broadening present at the considered time in the term associated with separate scatterings is reduced by the contribution coming from multiple collisions. Furthermore, this last contribution shifts the energy value of the peak towards the classical conservation energy. This effect can be better seen in Figure 3 (bottom), where the total distribution and the one for separate scatterings are plotted together, normalized to the same maximum value. We can conclude that the quantum correction including multiple scatterings is closer to the semiclassical result based on the assumption of completed collisions with respect to the quantum case where only separate scatterings are considered.

Summarizing, our results of the analysis of phonon scattering show that multiple collisions reduce collisional broadening and contribute to understand the success of the semiclassical approximation.

Simulation have been performed also for the case of scattering with a potential profile. At present, due to a still low efficiency of the numerical algorithm, it has been possible to obtain good quality results only for the case of very weak potentials.

Another interesting application of the method we are working on, is the study of the transient regime for bulk systems. Again the bottleneck is the efficiency of the algorithm that seriously limits the number of scatterings that can be accounted for. To improve such efficiency, a code including the quantum self-scattering mechanisms [11] is under development. This method is based on the introduction of an approximate immaginary selfenergy $\hbar / \tau_{0}$ which plays a role analogous to that of the maximum scattering rate in the traditional MC method. At each perturbative order, the exact correction to $1 / \tau_{0}$ is evaluated.

As a last point it should be mentioned that starting from the two-time $G^{<}$Green function it 
has been possible to define a general WF for one electron interacting with phonons, which is function of $p$ and $\omega$ separately, and which is defined as

$$
\begin{aligned}
& f_{W}(\mathbf{r}, \mathbf{p}, t, \omega)=-i \hbar G^{<}(\mathbf{r}, \mathbf{p}, t, \omega)=\sum_{\left\{n_{\mathbf{q}}\right\}} \int e^{i \mathbf{p}^{\prime} \mathbf{r}^{\prime} / \hbar} d \mathbf{r}^{\prime} \\
& \times \int e^{-i \omega \tau} \overline{g\left(\mathbf{r}-\mathbf{r}^{\prime} / 2,\left\{n_{\mathbf{q}}\right\}, t-\tau / 2\right) g^{*}\left(\mathbf{r}+\mathbf{r}^{\prime} / 2,\left\{n_{\mathbf{q}}^{\prime}\right\}, t+\tau / 2\right)} d \tau .
\end{aligned}
$$

where

$$
g\left(\mathbf{r},\left\{n_{\mathbf{q}}\right\}, t\right)=e^{i \omega\left(\left\{n_{\mathbf{q}}\right\}\right) t} \Phi\left(\mathbf{r},\left\{n_{\mathbf{q}}\right\}, t\right)
$$

$\Phi\left(\mathbf{r},\left\{n_{\mathbf{q}}\right\}, t\right)$ is the wave function of the system, and the bar means ensamble average. Equation (18) can be generalized to states non diagonal in the phonons variables:

$$
\begin{aligned}
& f_{W}\left(\mathbf{r}, \mathbf{p},\left\{n_{\mathbf{q}}\right\},\left\{n_{\mathbf{q}}^{\prime}\right\}, t, \omega\right)=\int e^{i \mathbf{p}^{\prime} \mathbf{r}^{\prime} / \hbar} d \mathbf{r}^{\prime} \\
& \times \int e^{-i \omega \tau} \overline{g\left(\mathbf{r}-\mathbf{r}^{\prime} / 2,\left\{n_{\mathbf{q}}\right\}, t-\tau / 2\right) g^{*}\left(\mathbf{r}+\mathbf{r}^{\prime} / 2,\left\{n_{\mathbf{q}}^{\prime}\right\}, t+\tau / 2\right)} d \tau .
\end{aligned}
$$

Performing the trace over the phonon variables of Eq. (20), for states diagonal in the phonon variables, the 1.h.s. of Eq. (18) is recovered. Details and preliminary results are shown in Ref. [12].

\section{PERSPECTIVES}

The leading idea in developing the concept of WP's has been to realize a tool for studying the transport properties of a mesoscopic semiconductor system, combining the rigorous quantum approach provided by the WF formalism with the high reliability, computational feasibility, and conceptual simplicity of a traditional MC program. At the moment, due to computational time problems, results have been obtained for time of the order of $100 \mathrm{fs}$, including only up to two phonon scatterings. Also the inclusion in the code of the scattering with the potential leads to such a computational burden that it has been impossible, so far, to simulate realistic potential profiles.
Therefore the short term goals of the approach described in this paper are: (i) to improve the efficiency of the numerical algorithm to make possible the inclusion in the simulation of a higher number of carrier-phonon scattering events, and (ii) the developments of analytical and numerical methods to make possible the inclusion in the simulation of the interaction of the carriers with realistic potential profiles. To this aim a parallel version of the quantum MC code will be implemented, suitable to exploit the features of modern supercomputers. Even more important, we belive that the efficiency of the algorithm should be strongly improved by the above mentioned introduction of the quantum self-scattering mechanism.

\section{Acknowledgements}

This work was partially supported by ONR (Contract N. N000149810777) and by the project 40\% 1996 sponsored by the Italian Ministry of University and Scientific and Technological Research.

\section{References}

[1] See, e.g., Sala, R., Brouard, S. and Muga, J. G. (1993). "Wigner trajectories and Liouville's theorem", J. Chem. Phys., 99, 2708.

[2] Lee, H.-W. and Scully, M. O. (1983). "The Wigner phasespace description of collision processes", Found. Phys., $13,61$.

[3] Pascoli, M., Bordone, P., Brunetti, R. and Jacoboni, C. (1998). "Wigner paths for electrons interacting with phonons", Phys. Rev. B, 58, 3503.

[4] Bertoni, A., Bordone, P., Brunetti, R. and Jacoboni, C. (1999). "The Wigner function for electron transport in mesoscopic systems", J. Phys.: Condensed Matter, 11, 5999.

[5] Rossi, F., Jacoboni, C. and Nedjalkov, M. (1994). "A Monte Carlo solution of the Wigner transport equation", Semicond. Sci. and Technol., 9, 934.

[6] Brunetti, R., Jacoboni, C. and Rossi, F. (1989). "Quantum theory of transient transport in semiconductors: a Monte Carlo approach", Phys. Rev. B, 39, 10781.

[7] Carruthers, P. and Zachariasen, F. (1983). "Quantum collision theory with phase-space distributions", Rev. Mod. Phys., 55, 245.

[8] Rees, H. D. (1972). "Numerical solution of electron motion in solids", J. Phys. C, 5, 64 . 
[9] Rossi, F., Poli, P. and Jacoboni, C. (1992). "The weighted Monte Carlo approach to electron transport in semiconductors", Semicond. Sci. Technol., 7, 1017.

[10] Bertoni, A., Bordone, P., Brunetti, R., Jacoboni, C. and Sano, N. (1999). "Quantum versus classical scattering in semiconductor charge transport: a quantitative comparison", Physica B, 272, 299.
[11] Rossi, F. and Jacoboni, C. (1992). "Self-scattering in Monte Carlo simulation of quantum transport", Europhys. Lett., 18, 169.

[12] Brunetti, R., Bertoni, A., Bordone, P. and Jacoboni, C. (2000). "Dynamical Equation and Monte Carlo Simulation of the Two-time Wigner Function for Electron Quantum Transport", VLSI Design, this volume. 

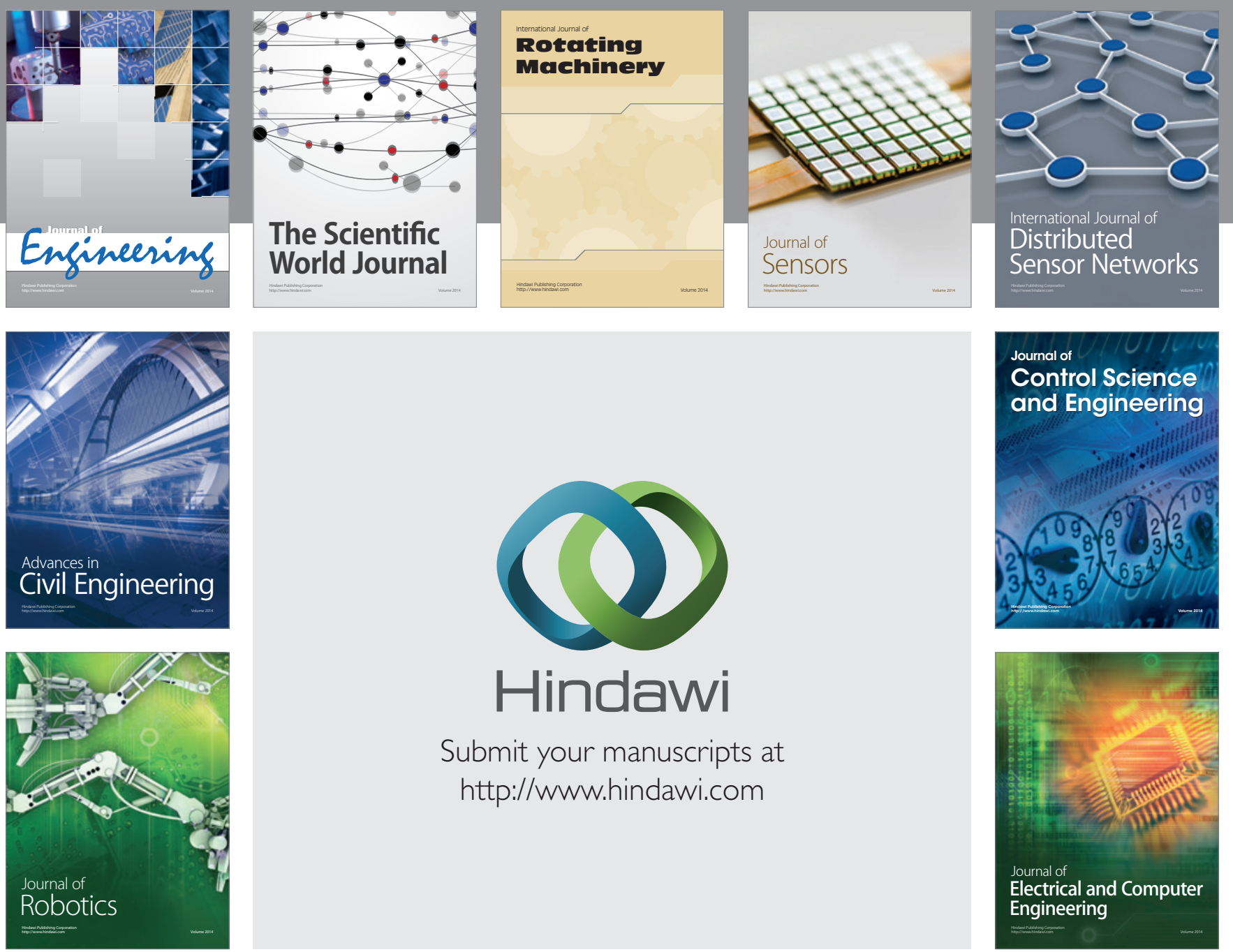

Submit your manuscripts at

http://www.hindawi.com
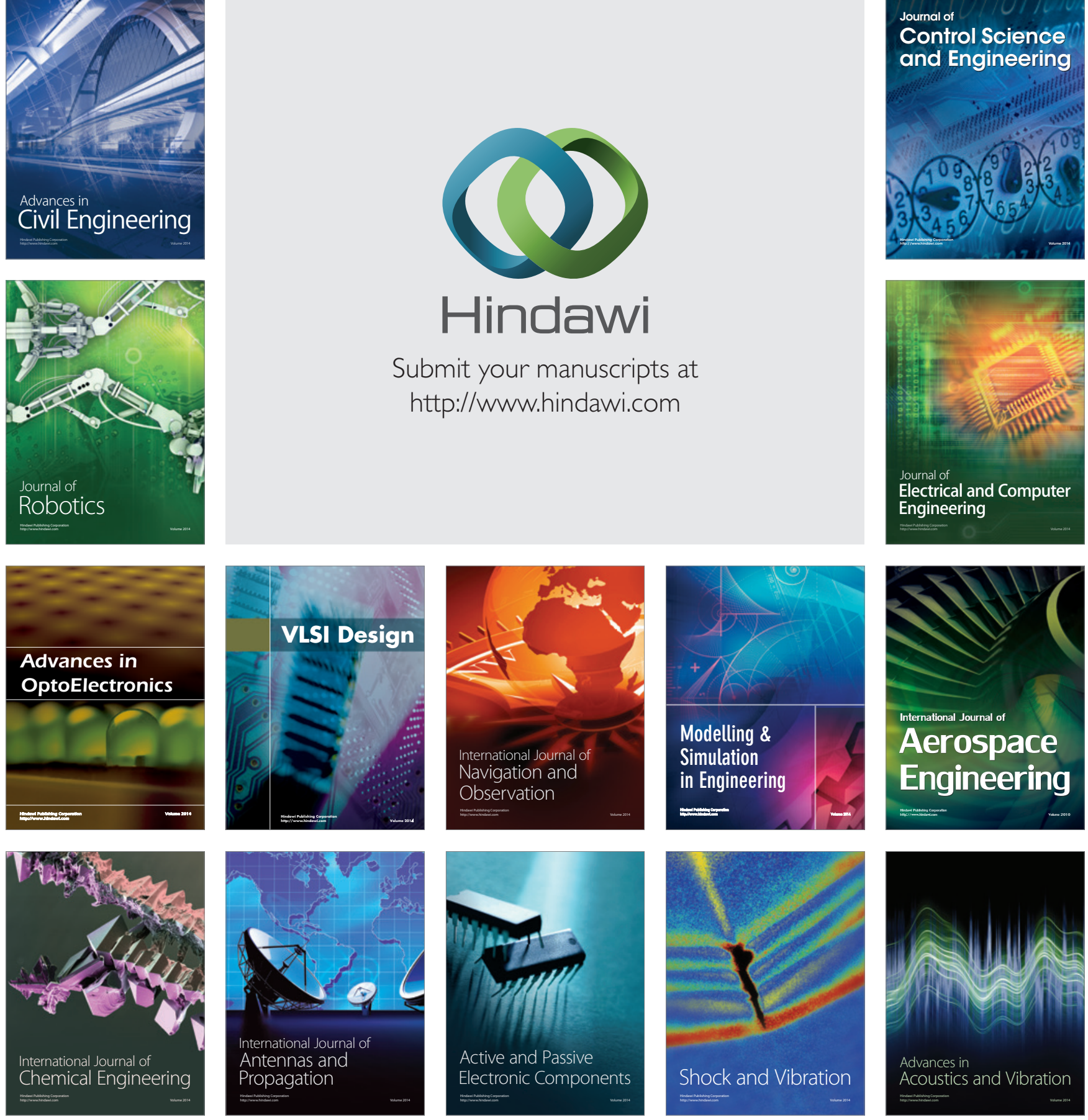